\title{
Is it safe to send home an uncomplicated diverticulitis? The DIVER trial
}

\author{
Ciro Paolillo • Ilenia Spallino
}

Received: 18 November 2014 / Accepted: 20 November 2014/Published online: 4 December 2014 (C) SIMI 2014

\section{Background}

Hospitalization, bowel rest, intravenous fluids and antibiotic therapy are universally accepted as the first choice treatment for mild diverticulitis [1]. Even if hospital admission is presently considered essential, the majority of patients do not develop a complicated disease and are treated medically, with fewer than $15 \%$ of patients requiring surgical intervention during the same admission [2]. For this reason, there is increasing interest in the ambulatory management of low-risk diverticulitis patients.

\section{Summary}

The Diver trial was a multicenter, 2-arm, parallel, 1:1, randomized non-inferiority trial [3]. The aim of the study was to determine the non-inferiority of home treatment vs hospitalization in the management of patients with uncomplicated left colonic diverticulitis.

Patients with acute uncomplicated diverticulitis were randomly allocated to hospitalization and usual treatment (group 1) or to outpatient management (group 2). In this case, they were discharged from the emergency department (ED), and called daily for 5 consecutive days by the study investigators.

All patients admitted to the ED with a clinical suspicion of diverticulitis underwent abdominal computed tomography (CT) with intravenous contrast administration. The

C. Paolillo $(\varangle) \cdot$ I. Spallino

SOC Medicina d'Urgenza e Ponto Soccorso AOU S.Maria della

Misericordia, Udine, UD, Italy

e-mail: ciropaolillo@gmail.com severity of diverticulitis was graded according to the modified Hinchey classification ("Appendix").

Patients were eligible if they were older than 18 years, with uncomplicated diverticulitis able to tolerate oral intake, and with good response to first treatment measures in the ED: improvement of pain and fever, and willing to continue treatment at home under supervision.

Exclusion criteria were complicated colonic diverticulitis, absence of symptom relief, pregnancy or breastfeeding, antibiotic treatment for colonic diverticulitis in the previous month, colorectal cancer suspicion on the CT scan, concomitant unstable comorbid conditions, immunosuppression, cognitive, social, or psychiatric impairment, intolerance to oral intake and persisting vomiting, or patients' rejection of written consent.

The primary end point of the trial was the treatment failure rate, defined as the persistence, increase, or recurrence of abdominal pain or fever, inflammatory bowel obstruction, need for radiological abscess drainage or immediate surgery due to complicated diverticulitis, need for hospital admission, and mortality during the first 60 days after discharge.

Secondary end points included a quality-of-life assessment at days 14 and 60 after discharge, and the evaluation of costs for both management strategies.

To evaluate the primary end point, a $10 \%$ non-inferiority boundary was used. Data were analyzed by intentionto-treat.

All patients in the study protocol were randomized to hospitalization (group 1) or to outpatient management (group 2). The first dose of antibiotic treatment was given intravenously to all patients of both groups in the ED. Antibiotic treatment in both arms was discontinued after 10 days. 
Seven patients were readmitted because of treatment failure: 4 patients $(6.1 \%)$ in group 1 and 3 patients $(4.5 \%)$ in group 2. No differences were observed between the two groups $(P=0.619)$. No patients needed emergency surgery as a consequence of readmission, and no death was observed.

The quality of life was similar in both groups at 14 and 60 days. The overall healthcare cost per episode was 3 times lower in group 2 as compared to group 1 .

\section{Strength of the study}

- It is the first multicenter randomized clinical trial dealing with this question.

\section{Weaknesses of the study}

- A high number of patients were not suitable for randomization. Indeed, of the 453 patients treated for colonic diverticulitis, 258 were eligible and only 132 were randomized. This could affect external validity.

- Even if the trial is designed as a non-inferiority study, data were analyzed and reported as a superiority trial. This creates some confusion and makes the results less clear.

\section{Question marks}

- The number of severe adverse events in uncomplicated diverticulitis is low, so the authors decided to test some surrogate and subjective end points, as the persistence of pain. Since the outcomes included in the composite primary end point are not equally clinically important, it would be interesting to know the exact reasons for treatment failure in the two groups.

- We wonder if the study protocol is easily applicable in everyday clinical practice. Indeed, some procedures used in the study, as the need for abdominal CT scan in every patient to assign a Hinchey score or the daily phone monitoring, might be difficult to perform.

- As in every non-inferiority trial, the choice of the noninferiority boundary is crucial. We wonder if in this case a $10 \%$ difference in the primary end point is clinically relevant.

- Since CT scan results were part of the inclusion criteria, we wonder how good was the concordance between CT scan interpretations by different radiologists.

\section{Sponsorship}

The trial was supported by a grant by the Instituto Carlos III from the Ministry of Health, Government of Spain.

\section{Clinical bottom line}

Serious adverse events in uncomplicated diverticulitis are rare, and outpatient management could be selected as an option. Evidence that all patients could be treated as outpatients is too scanty, and the decision should still be individualized in each case.

Conflict of interest None.

\section{Appendix}

Hynchey modified classification

Stage 0 Mild clinical diverticulitis

Ia Confined pericolic inflammation-phlegmon

Ib Confined pericolic abscess

II Pelvic, intra-abdominal, or retroperitoneal abscesses III Generalized purulent peritonitis

IV Generalized fecal peritonitis

\section{References}

1. Touzios JG et al (2009) Diverticulosis and acute diverticulitis. Gastronterol Clin North Am 38:513-525

2. Mizuki A et al (2005) The outpatient management of patients with acute mild-to-moderate colonic diverticulitis. Aliment Pharmacol Ther 21:889-897

3. Biondo S et al (2014) Outpatient versus hospitalization management for uncomplicated diverticulitis A prospective multi center randomized clinical trial (DIVER trial). Ann Surg 259:1 